\title{
Trial of Everolimus-Eluting Stents or Bypass Surgery for Coronary Disease
}

\author{
Seung-Jung Park, M.D., Ph.D., Jung-Min Ahn, M.D., Young-Hak Kim, M.D., \\ Duk-Woo Park, M.D., Sung-Cheol Yun, Ph.D., Jong-Young Lee, M.D., \\ Soo-Jin Kang, M.D., Seung-Whan Lee, M.D., Cheol Whan Lee, M.D., \\ Seong-Wook Park, M.D., Suk Jung Choo, M.D., Cheol Hyun Chung, M.D., \\ Jae Won Lee, M.D., David J. Cohen, M.D., Alan C. Yeung, M.D., Seung Ho Hur, M.D., \\ Ki Bae Seung, M.D., Tae Hoon Ahn, M.D., Hyuck Moon Kwon, M.D., \\ Do-Sun Lim, M.D., Seung-Woon Rha, M.D., Myung-HoJeong, M.D., Bong-Ki Lee, M.D., \\ Damras Tresukosol, M.D., Guo Sheng Fu, M.D., and Tiong Kiam Ong, M.D., \\ for the BEST Trial Investigators*
}

From the Heart Institute (S.-J.P., J.-M.A. Y.-H.K., D.-W.P., J.-Y.L., S.-J.K., S.-W.L., C.W.L., S.-W.P., S.J.C., C.H.C., J.W.L.) and Division of Biostatistics (S.-C.Y.), Center for Medical Research and Information, University of Ulsan College of Medicine, Asan Medical Center, Catholic University of Korea, St. Mary's Hospital (K.B.S.), Gangnam Severance Hospital (H.M.K.), Korea University Anam (D.-S.L.), and Guro Hospital (S.-W.R.), Seoul, Keimyung University Dongsan Medical Center, Daegu (S.H.H.), Gachon University Gil Hospital, Incheon (T.H.A.), Chonnam National University Hospital, Gwangju (M.-H.J.), and Kangwon National University Hospital, Chuncheon (B.-K.L.) — all in South Korea; Saint Luke's Mid America Heart Institute, University of Missouri-Kansas City, Kansas City (D.J.C.); Stanford University School of Medicine, Palo Alto, CA (A.C.Y.); Siriraj Hospital, Bangkok, Thailand (D.T.); Sir Run Run Shaw Hospital, Hangzhou, China (G.S.F.); and Sarawak General Hospital, Kuching, Malaysia (T.K.O.). Address reprint requests to Dr. S.-J. Park at the Heart Institute, Asan Medical Center, University of Ulsan, 388-1 Pungnap-dong, Songpa-gu, Seoul, 138-736, South Korea, or atsjpark@amc.seoul.kr.

Drs. S.-J. Park and J.-M. Ahn contributed equally to this article.

*A complete list of investigators, institutions, and research organizations participating in the Randomized Comparison of Coronary Artery Bypass Surgery and Everolimus-Eluting Stent Implantation in the Treatment of Patients with Multivessel Coronary Artery Disease (BEST) trial is provided in the Supplementary Appendix, available at NEJM.org.

This article was published on March 16, 2015, at NEJM.org.

N Engl J Med 2015;372:1204-12.

DOI: 10.1056/NEJMoal415447

Copyright (c) 2015 Massachusetts Medical Society.

\section{ABSTRACT}

\section{BACKGROUND}

Most trials comparing percutaneous coronary intervention (PCI) with coronaryartery bypass grafting (CABG) have not made use of second-generation drug-eluting stents.

\section{METHODS}

We conducted a randomized noninferiority trial at 27 centers in East Asia. We planned to randomly assign 1776 patients with multivessel coronary artery disease to PCI with everolimus-eluting stents or to CABG. The primary end point was a composite of death, myocardial infarction, or target-vessel revascularization at 2 years after randomization. Event rates during longer-term follow-up were also compared between groups.

\section{RESULTS}

After the enrollment of 880 patients (438 patients randomly assigned to the PCI group and 442 randomly assigned to the CABG group), the study was terminated early owing to slow enrollment. At 2 years, the primary end point had occurred in $11.0 \%$ of the patients in the PCI group and in $7.9 \%$ of those in the CABG group (absolute risk difference, 3.1 percentage points; 95\% confidence interval [CI], -0.8 to 6.9; $\mathrm{P}=0.32$ for noninferiority). At longer-term follow-up (median, 4.6 years), the primary end point had occurred in $15.3 \%$ of the patients in the PCI group and in $10.6 \%$ of those in the CABG group (hazard ratio, $1.47 ; 95 \% \mathrm{CI}, 1.01$ to $2.13 ; \mathrm{P}=0.04$ ). No significant differences were seen between the two groups in the occurrence of a composite safety end point of death, myocardial infarction, or stroke. However, the rates of any repeat revascularization and spontaneous myocardial infarction were significantly higher after PCI than after CABG.

\section{CONCLUSIONS}

Among patients with multivessel coronary artery disease, the rate of major adverse cardiovascular events was higher among those who had undergone PCI with the use of everolimus-eluting stents than among those who had undergone CABG. (Funded by CardioVascular Research Foundation and others; BEST ClinicalTrials.gov number, NCT00997828.) 
ANDOMIZED TRIALS AND OBSERVATIONal studies have shown that the rates of most adverse clinical outcomes among patients with multivessel coronary artery disease are lower after coronary-artery bypass grafting (CABG) than after percutaneous coronary intervention (PCI). ${ }^{1-7}$ Current clinical guidelines thus recommend $\mathrm{CABG}$ as the preferred revascularization strategy, particularly in patients with complex coronary lesions and without excessive operative risk. ${ }^{8,9}$ However, previous trials may have been limited by their use of first-generation drug-eluting stents. Although these stents reduced the rate of restenosis, their use was associated with a relatively high rate of stent-related thrombotic events. ${ }^{10}$ Results from the Synergy between PCI with Taxus and Cardiac Surgery (SYNTAX) trial showed, for example, that approximately one fourth of the clinical events occurring in the PCI group were associated with stent thrombosis. ${ }^{11}$

Over the past decade, second-generation drugeluting stents have improved outcomes with PCI significantly. Randomized trials and meta-analyses have shown that the use of everolimus-eluting stents markedly reduces the rates of death, myocardial infarction, restenosis, and stent thrombosis, suggesting that everolimus-eluting stents are safer and more effective than firstgeneration drug-eluting stents. ${ }^{12,13}$ The Randomized Comparison of Coronary Artery Bypass Surgery and Everolimus-Eluting Stent Implantation in the Treatment of Patients with Multivessel Coronary Artery Disease (BEST) trial was designed to compare the outcomes in patients with multivessel coronary artery disease who have undergone PCI with the use of everolimuseluting stents with the outcomes in those who have undergone CABG.

\section{METHODS}

\section{STUDY DESIGN AND PATIENTS}

The BEST trial was a prospective, open-label, randomized trial that was conducted at 27 sites in South Korea, China, Malaysia, and Thailand. The trial was designed by the first author, and the protocol (available with the full text of this article at NEJM.org) was approved by the institutional review board at each participating center. The funders assisted in the design of the protocol but had no role in the conduct of the trial or in the analysis or interpretation of the data. The first author had unrestricted access to the data after the database was locked and prepared all drafts of the manuscript with input from the other authors. The first author vouches for the completeness and accuracy of the data and the analyses, as well as for the fidelity of the study to the trial protocol.

Eligible patients were 18 years of age or older, had angiographically confirmed multivessel coronary artery disease with stenoses of more than $70 \%$ of the vessel diameter in major epicardial vessels in the territories of at least two coronary arteries, and were considered by the physicians and surgeons who were treating them to be suitable candidates for either PCI or CABG. Patients with clinically significant left main coronary artery disease were excluded. A complete list of inclusion and exclusion criteria is provided in the Supplementary Appendix, available at NEJM.org. All the study participants provided written informed consent.

\section{STUDY PROCEDURES AND FOLLOW-UP}

We enrolled the study patients after diagnostic coronary angiography was performed. Eligible patients were randomly assigned in a 1:1 ratio, with the use of an interactive Web-response system, to undergo PCI with the use of everolimuseluting stents or to undergo CABG. Randomization was computer-generated and was performed in random block sizes of 6 and 8, with stratification according to the participating center.

The procedures for PCI and CABG have been described previously. ${ }^{8,14}$ During PCI, we attempted to treat all lesions with everolimus-eluting stents. The use of intravascular ultrasonography, adjunctive devices, or glycoprotein IIb/IIIa inhibitors was at the physician's discretion. All the patients undergoing PCI were prescribed aspirin plus clopidogrel before or during the procedure. After PCI, all the patients received aspirin at a dose of $100 \mathrm{mg}$ per day indefinitely and clopidogrel at a dose of $75 \mathrm{mg}$ per day for at least 12 months.

During CABG, the internal thoracic artery was used preferentially for revascularization of the left anterior descending coronary artery. The medications administered after CABG were selected according to the policy of the institution or physician. Throughout the study period, the use of secondary-prevention medication according to clinical guidelines was strongly recommended, and the importance of lifestyle modification, 
such as smoking cessation and improvements in nutrition and exercise habits, was also emphasized.

Routine angiographic follow-up was strongly discouraged for all the patients in order to reduce the occurrence of repeat revascularization driven by angiographic findings alone in the absence of signs or symptoms of ischemia. Immediate follow-up assessments were performed at each hospital, and follow-up assessments were performed by means of clinic visits or telephone interviews at 30 days and at 6,9 , and 12 months, and annually thereafter.

\section{END POINTS}

The primary end point was a composite of death, myocardial infarction, or target-vessel revascularization. Major secondary end points were a safety composite of death, myocardial infarction, or stroke and a composite of death, myocardial infarction, stroke, or any repeat revascularization. Additional secondary end points included the individual components of the composite end points as well as stent thrombosis and major or fatal bleeding. Detailed definitions of the trial end points are provided in the Supplementary Appendix. The extent of revascularization was recorded by the investigators, with complete revascularization defined as revascularization of all diseased segments that were at least $2.0 \mathrm{~mm}$ in diameter.

All the clinical end points were assessed by the event-adjudication committee, whose members were unaware of the study-group assignments. All angiographic data were analyzed in the angiographic core laboratory of the CardioVascular Research Foundation, Seoul, South Korea. ${ }^{14}$ Data quality was monitored systematically as described in the Supplementary Appendix.

\section{STATISTICAL ANALYSIS}

The study was designed as a noninferiority trial. Assuming a $12 \%$ incidence of the primary end point at 2 years with $\mathrm{CABG}^{15,16}$ and using a noninferiority margin of 4\%, we estimated that 1776 patients would need to undergo randomization in order for the study to have $80 \%$ power to show the noninferiority of PCI with everolimus-eluting stents. However, the enrollment rate was slower than expected, which was thought to be a consequence of the rapid increase in the use of measurement of fractional flow reserve in clinical practice.

In October 2013, by which time 880 patients had been enrolled, the data and safety monitoring board recommended stopping enrollment. The decision-making process for the premature termination of the trial is described in the Supplementary Appendix. The 2-year analysis of the primary end point prespecified in the trial protocol was supplemented by exploratory analyses of longer-term outcomes with the use of all available follow-up data. All the analyses were performed according to the intention-to-treat principle, except as noted.

Baseline clinical and angiographic characteristics and procedural data were compared in the two trial groups with the use of Student's t-test for continuous variables and the chi-square test or Fisher's exact test for categorical variables, as appropriate. Survival was assessed with the use of the Kaplan-Meier method and compared with the use of the log-rank test. Event rates of clinical end points were compared with the use of the log-rank test for the time to the first event after randomization. Hazard ratios and 95\% confidence intervals were estimated with the use of Cox proportional-hazard models. The proportional-hazards assumption regarding the treatment assignments was confirmed by means of the Schoenfeld residuals test; no relevant violations of the assumption were found except in the case of myocardial infarction. ${ }^{17}$ We also performed separate landmark analyses using a cutoff point of 30 days after randomization, with hazard ratios calculated separately for events that occurred within 30 days and those that occurred after 30 days. The consistency of treatment effects in subgroups was assessed with the use of Cox regression models with tests for interaction. All $\mathrm{P}$ values and $95 \%$ confidence intervals were two-sided. SAS software, version 9.3 (SAS Institute), was used for all the statistical analyses.

\section{RESULTS}

\section{STUDY POPULATION}

From July 2008 through September 2013, a total of 4654 patients were screened for enrollment in this study (Fig. S1 and Table S1 in the Supplementary Appendix). Of the 1725 eligible patients, 880 provided written informed consent and were randomly assigned to undergo PCI with everolimus-eluting stents (438 patients) or CABG (442). A total of 413 patients assigned to PCI and 382 assigned to CABG received the assigned treatment (Fig. S1 in the Supplementary Appendix). 
The demographic, clinical, and angiographic characteristics of the patients in the two groups were well matched at baseline (Table 1).

Patients in the PCI group received an average of 3.4 stents per patient, with intravascular ultrasonography used in $71.8 \%$ of the patients during PCI. In the CABG group, $64.3 \%$ of the patients underwent off-pump surgery, and $99.3 \%$ underwent revascularization of the left anterior descending coronary artery with the left internal thoracic artery. Complete revascularization occurred more frequently in the CABG group than in the PCI group $(71.5 \%$ vs. $50.9 \%, \mathrm{P}<0.001)$ (Table 2).

Medical management at discharge and followup differed between the PCI and CABG groups. Patients in the PCI group were significantly more likely to receive certain medications, including antiplatelet agents, beta-blockers, angiotensin-converting-enzyme inhibitors or angiotensin II-receptor blockers, and calcium-channel blockers (Table S2 in the Supplementary Appendix).

\section{FOLLOW-UP}

A total of 3 patients ( 1 in the PCI group and 2 in the CABG group) were lost to follow-up (Fig. S1 in the Supplementary Appendix). Among survivors, the median length of follow-up after randomization was 4.6 years (interquartile range, 3.5 to 5.2), with no significant between-group difference $(\mathrm{P}=0.94)$. Routine angiographic follow-up was performed in 48 patients $(11.0 \%)$ in the PCI group and in $16(3.6 \%)$ in the CABG group $(\mathrm{P}<0.001)$.

\section{PRIMARY END POINT}

At 2 years, the primary end point of death, myocardial infarction, or target-vessel revascularization had occurred in 48 patients $(11.0 \%)$ who had been randomly assigned to PCI and in 35 (7.9\%) who had been randomly assigned to CABG (absolute risk difference, 3.1 percentage points; $95 \%$ confidence interval [CI], -0.8 to $6.9 ; \mathrm{P}=0.32$ for noninferiority). In an as-treated analysis, the 2-year rates of the primary end point were $11.2 \%$ and $7.5 \%$, respectively (absolute risk difference, 3.7 percentage points; 95\% CI, -0.2 to 7.6 ; $\mathrm{P}=0.44$ for noninferiority).

During long-term follow-up, the primary end point occurred more frequently in the PCI group than in the CABG group (15.3\% vs. $10.6 \%$; hazard ratio, 1.47; 95\% CI, 1.01 to $2.13 ; \mathrm{P}=0.04$ ) (Fig. 1A and Table 3). The as-treated analysis
Table 1. Characteristics of the Patients at Baseline, According to Study Group.*

\begin{tabular}{|c|c|c|}
\hline Characteristic & $\begin{array}{c}\mathrm{PCl} \\
(\mathrm{N}=438)\end{array}$ & $\begin{array}{c}\text { CABG } \\
(\mathrm{N}=442)\end{array}$ \\
\hline Age $-y r$ & $64.0 \pm 9.3$ & $64.9 \pm 9.4$ \\
\hline Male sex - no. (\%) & $304(69.4)$ & $325(73.5)$ \\
\hline Body-mass index $†$ & $24.7 \pm 2.9$ & $25.0 \pm 2.9$ \\
\hline \multicolumn{3}{|l|}{ Medically treated diabetes - no. (\%) } \\
\hline Any & $177(40.4)$ & $186(42.1)$ \\
\hline Requiring insulin & $20(4.6)$ & $18(4.1)$ \\
\hline Hypertension - no. (\%) & $296(67.6)$ & $295(66.7)$ \\
\hline Hyperlipidemia — no. (\%) & $239(54.6)$ & $222(50.2)$ \\
\hline Current smoker - no. (\%) & $88(20.1)$ & $89(20.1)$ \\
\hline Previous $\mathrm{PCl}$ — no. (\%) & $30(6.8)$ & $38(8.6)$ \\
\hline Previous myocardial infarction — no. (\%) & $25(5.7)$ & $29(6.6)$ \\
\hline Previous congestive heart failure - no. (\%) & $16(3.7)$ & $12(2.7)$ \\
\hline Previous stroke - no. (\%) & $37(8.4)$ & $33(7.5)$ \\
\hline Chronic renal failure — no. (\%) & $9(2.1)$ & $7(1.6)$ \\
\hline Peripheral vascular disease — no. (\%) & $15(3.4)$ & $12(2.7)$ \\
\hline $\begin{array}{l}\text { Chronic obstructive pulmonary disease - } \\
\text { no. (\%) }\end{array}$ & $8(1.8)$ & $6(1.4)$ \\
\hline \multicolumn{3}{|l|}{ Clinical presentation — no. (\%) } \\
\hline Stable angina & $210(47.9)$ & $204(46.2)$ \\
\hline Unstable angina & $185(42.2)$ & $199(45.0)$ \\
\hline $\begin{array}{l}\text { Acute myocardial infarction } \leq 90 \text { days } \\
\text { previously }\end{array}$ & $43(9.8)$ & $39(8.8)$ \\
\hline Ejection fraction - \% & $59.1 \pm 8.5$ & $59.9 \pm 8.1$ \\
\hline \multicolumn{3}{|l|}{ No. of diseased vessels — no. (\%) } \\
\hline 3 & $330(75.3)$ & $349(79.0)$ \\
\hline 2 & $108(24.7)$ & $93(21.0)$ \\
\hline Chronic total occlusion — no. (\%) & $126(28.8)$ & $138(31.2)$ \\
\hline Bifurcation — no. (\%) & $252(57.5)$ & $260(58.8)$ \\
\hline Heavily calcified lesion — no. (\%) & $141(32.2)$ & $134(30.3)$ \\
\hline \multicolumn{3}{|l|}{ EuroSCORE } \\
\hline Mean score & $2.9 \pm 2.0$ & $3.0 \pm 2.1$ \\
\hline$\geq 6-$ no. (\%) & $51(11.6)$ & $59(13.3)$ \\
\hline \multicolumn{3}{|l|}{ SYNTAX score $\mathbb{E}$} \\
\hline Mean score & $24.2 \pm 7.5$ & $24.6 \pm 8.1$ \\
\hline$\geq 33-$ no. (\%) & $66(15.1)$ & 79 (17.9) \\
\hline
\end{tabular}

* Plus-minus values are means \pm SD. Data are shown for the intention-to-treat population. There were no significant differences between groups in the comparisons of baseline characteristics. CABG denotes coronary-artery bypass grafting, and $\mathrm{PCl}$ percutaneous coronary intervention.

$\uparrow$ The body-mass index is the weight in kilograms divided by the square of the height in meters.

$¥$ The European System for Cardiac Operative Risk Evaluation (EuroSCORE) is a clinical model for calculating the risk of death after cardiac surgery. Scores range from 0 to 39, with higher scores indicating greater risk. A score of 6 or more indicates high operative risk.

$\int$ The Synergy between PCI with Taxus and Cardiac Surgery (SYNTAX) score is an angiographic model for evaluating the extensiveness of coronary artery disease. Scores range from 0 to 115 , with higher scores indicating more complex disease. A score of 33 or more indicates high complexity of coronary disease. 


\begin{tabular}{|lc|}
\hline \multicolumn{2}{|l|}{ Table 2. Procedural Characteristics of the Patients, According to Study Group.* } \\
\hline Characteristic & Value \\
PCI group & 464 \\
No. of patients & $3.4 \pm 1.4$ \\
No. of stents placed & $85.3 \pm 38.2$ \\
Total length of stents placed - mm & $3.1 \pm 0.3$ \\
Stent diameter — mm & $333(71.8)$ \\
Intravascular ultrasonography - no. (\%) & $236(50.9)$ \\
Complete revascularization - no. (\%) $\dagger$ & \\
CABG group & 401 \\
No. of patients & \\
No. of grafted vessels per patient & $3.1 \pm 0.9$ \\
$\quad$ Any & $2.1 \pm 1.1$ \\
$\quad$ Arterial graft & $1.0 \pm 0.8$ \\
$\quad$ Vein graft & $398(99.3)$ \\
Left internal thoracic artery graft — no. (\%) & $258(64.3)$ \\
Off-pump surgery - no. (\%) & $274 / 383(71.5)$ \\
Complete revascularization — no./total no. (\%) $†$ & \\
\hline
\end{tabular}

* Plus-minus values are means \pm SD. Data were summarized according to the as-treated analysis.

$\uparrow \mathrm{P}<0.001$ for the comparison of $\mathrm{PCI}$ with CABG. Complete revascularization was defined as revascularization in all diseased segments that were at least $2.0 \mathrm{~mm}$ in diameter; information on complete revascularization was recorded by the investigators.
$2.9 \%$, respectively; $\mathrm{P}=0.72$ ) and myocardial infarction $(4.8 \%$ and $2.7 \%$, respectively; $\mathrm{P}=0.11)$. However, the rate of spontaneous myocardial infarction was significantly higher among patients who had undergone PCI than among those who had undergone CABG $(4.3 \%$ vs. $1.6 \%$, $\mathrm{P}=0.02$ ). Therefore, in the landmark analysis (Fig. S3 in the Supplementary Appendix) of events that occurred more than 30 days after randomization, there were more patients with myocardial infarction in the PCI group than in the CABG group ( $3.5 \%$ vs. $0.7 \%, \mathrm{P}=0.004$ ).

The rate of any repeat revascularization was significantly higher in the PCI group than in the CABG group ( $11.0 \%$ vs. $5.4 \%, \mathrm{P}=0.003$ ) (Table 3 , and Fig. S4 in the Supplementary Appendix). The rates of target-vessel revascularization and newlesion revascularization were also significantly higher with PCI than with CABG. These findings were consistent in the as-treated analyses (Table S3 in the Supplementary Appendix).

Major bleeding, according to the Thrombolysis in Myocardial Infarction (TIMI) definition, ${ }^{18}$ occurred less frequently in the PCI group than in the CABG group (30 patients [6.8\%] vs. 132 [29.9\%], $\mathrm{P}<0.001)$, mostly as a consequence of bleeding related to the CABG procedure (which occurred in 125 patients). The rate of fatal bleeding did not differ significantly between the groups (Table 3).

Stent thrombosis, according to the Academic Research Consortium classification, ${ }^{19}$ occurred in seven patients $(1.6 \%$, according to the intention-to-treat analysis) after index PCI; four patients had definite stent thrombosis, and three had probable stent thrombosis. Of the four patients with definite stent thrombosis, one had a fatal myocardial infarction.

\section{SUBGROUP ANALYSES}

The effect of treatment assignment on the primary end point was consistent across subgroups except with respect to diabetes status, in which a trend toward a treatment-by-subgroup interaction was found (Fig. 2). Among patients with diabetes, the rate of the primary end point was significantly higher among those assigned to PCI than among those assigned to CABG (19.2\% vs. $9.1 \%, \mathrm{P}=0.007)$. Among patients without diabetes, there was no significant difference in the rate of the primary end point between the PCI group and the CABG group $(12.6 \%$ and $11.7 \%$, 
respectively; $\mathrm{P}=0.79$ ) ( $\mathrm{P}=0.06$ for interaction). The interaction between treatment group and diabetes status was also observed with respect to the end points of any repeat revascularization $(\mathrm{P}=0.04$ for interaction) and the composite of death, myocardial infarction, stroke, or any repeat revascularization ( $\mathrm{P}=0.05$ for interaction) (Fig. S5 and S6 in the Supplementary Appendix).

\section{DISCUSSION}

In the BEST trial, PCI with everolimus-eluting stents was not shown to be noninferior to CABG with respect to the primary end point of death, myocardial infarction, or target-vessel revascularization at 2 years. At longer-term follow-up (median, 4.6 years), PCI was associated with a significant increase in the incidence of the primary end point, as compared with the incidence with CABG. This difference was related mainly to the higher rate of target-vessel revascularization in the PCI group. Although the rate of the composite safety end point of death, myocardial infarction, or stroke did not differ significantly between the two groups, the rates of spontaneous myocardial infarction and new-lesion revascularization were greater with PCI than with CABG - differences that emerged early and continued to increase throughout the follow-up period. The observed increases in repeat revascularization and spontaneous myocardial infarction with PCI did not appear to translate into an overall increase in mortality, although the power to detect a difference in mortality was limited; longer-term follow-up may help to determine whether these findings are durable.

With regard to the rate of stroke, we found no significant difference between the PCI group and the CABG group, a finding that contrasts with the results of previous randomized trials and a recent meta-analysis. ${ }^{1,4,20}$ The reason for this discrepancy is not clear, but the use of offpump CABG can avoid excessive manipulation of the aorta and may have contributed to a reduced rate of stroke in the CABG group in our study. ${ }^{21}$ The low incidence of ascending-aorta or aortic-arch calcification in the Asian population in general may also underlie a reduced rate of procedure-related stroke. ${ }^{22}$ Furthermore, the power of our study to show a difference in the rate of stroke was limited.

Patients who have diabetes and advanced

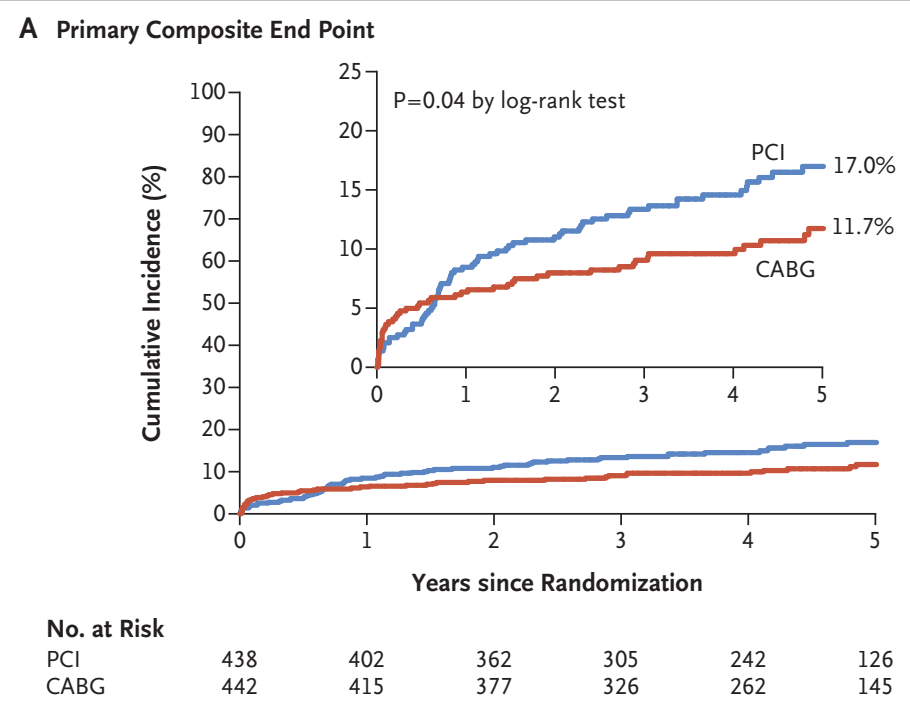

B Death, Myocardial Infarction, Stroke, or Repeat Revascularization

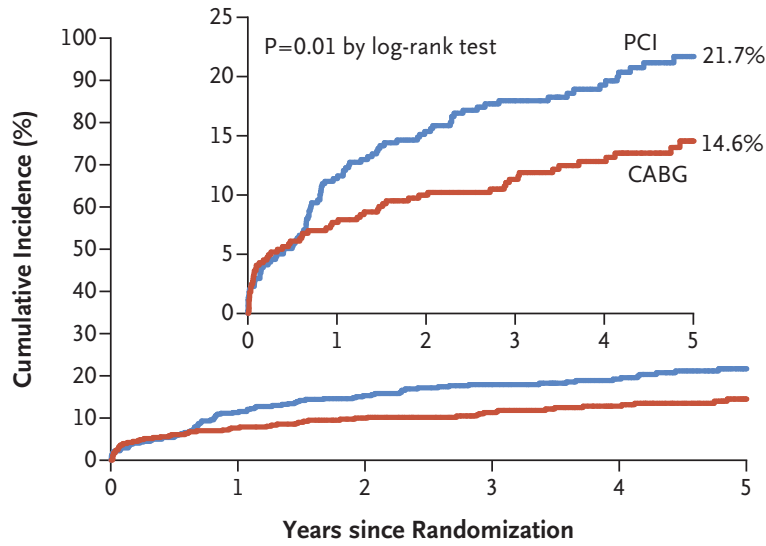

$\begin{array}{lllllll}\text { No. at Risk } & & & & & & \\ \text { PCI } & 438 & 389 & 341 & 288 & 229 & 117 \\ \text { CABG } & 442 & 409 & 368 & 317 & 250 & 137\end{array}$

Figure 1. Kaplan-Meier Curves for the Primary End Point and the Major Secondary End Point.

Panel A shows the cumulative incidence of the primary composite end point of death, myocardial infarction, or target-vessel revascularization, and Panel B the cumulative incidence of the secondary composite end point of death, myocardial infarction, stroke, or any repeat revascularization after randomization. Event rates shown are Kaplan-Meier estimates. The $P$ value was calculated by means of the log-rank test on the basis of all available follow-up data. The insets show the same data on enlarged y axes. CABG denotes coronary-artery bypass grafting, and $\mathrm{PCI}$ percutaneous coronary intervention.

coronary artery disease have been reported to have better outcomes with CABG than with PCI. $4,23,24$ We found a trend toward an interaction between revascularization type and diabetes 


\begin{tabular}{|c|c|c|c|c|}
\hline End Point & $\begin{array}{c}\mathrm{PCl} \\
(\mathrm{N}=438)\end{array}$ & $\begin{array}{l}\text { CABG } \\
(\mathrm{N}=442)\end{array}$ & $\begin{array}{l}\text { Hazard Ratio } \\
(95 \% \mathrm{Cl}) \div\end{array}$ & P Value \\
\hline \multicolumn{5}{|c|}{ number (percent) } \\
\hline $\begin{array}{c}\text { Primary end point: death, myocardial infarction, } \\
\text { or target-vessel revascularization }\end{array}$ & $67(15.3)$ & $47(10.6)$ & $1.47(1.01-2.13)$ & 0.04 \\
\hline \multicolumn{5}{|l|}{ Secondary end points } \\
\hline \multicolumn{5}{|l|}{ Death } \\
\hline Any cause & $29(6.6)$ & $22(5.0)$ & $1.34(0.77-2.34)$ & 0.30 \\
\hline Cardiac cause & $18(4.1)$ & $16(3.6)$ & $1.15(0.58-2.25)$ & 0.69 \\
\hline Noncardiac cause & $11(2.5)$ & $6(1.4)$ & $1.87(0.69-5.05)$ & 0.21 \\
\hline \multicolumn{5}{|l|}{ Myocardial infarction } \\
\hline Any & $21(4.8)$ & $12(2.7)$ & $1.76(0.87-3.58)$ & 0.11 \\
\hline Fatal & $4(0.9)$ & 0 & NA & NA \\
\hline Spontaneous & $19(4.3)$ & $7(1.6)$ & $2.75(1.16-6.54)$ & 0.02 \\
\hline Spontaneous Q wave & $4(0.9)$ & $2(0.5)$ & $2.03(0.37-11.1)$ & 0.40 \\
\hline Death or myocardial infarction & $43(9.8)$ & $34(7.7)$ & $1.28(0.82-2.01)$ & 0.28 \\
\hline \multicolumn{5}{|l|}{ Stroke } \\
\hline Any & $11(2.5)$ & $13(2.9)$ & $0.86(0.39-1.93)$ & 0.72 \\
\hline Ischemic stroke & $9(2.1)$ & $12(2.7)$ & $0.77(0.32-1.82)$ & 0.54 \\
\hline Hemorrhagic stroke & $2(0.5)$ & $1(0.2)$ & $2.03(0.18-22.4)$ & 0.55 \\
\hline Death, myocardial infarction, or stroke & $52(11.9)$ & $42(9.5)$ & $1.26(0.84-1.89)$ & 0.26 \\
\hline $\begin{array}{l}\text { Death from cardiac cause, myocardial } \\
\text { infarction, or stroke }\end{array}$ & $42(9.6)$ & $37(8.4)$ & $1.16(0.74-1.80)$ & 0.52 \\
\hline \multicolumn{5}{|l|}{ Repeat revascularization } \\
\hline Any & $48(11.0)$ & $24(5.4)$ & $2.09(1.28-3.41)$ & 0.003 \\
\hline Target vessel & $31(7.1)$ & $17(3.8)$ & $1.88(1.04-3.40)$ & 0.03 \\
\hline Target lesion & $25(5.7)$ & $17(3.8)$ & $1.51(0.82-2.80)$ & 0.19 \\
\hline New lesion & $24(5.5)$ & $10(2.3)$ & $2.47(1.18-5.17)$ & 0.01 \\
\hline $\begin{array}{l}\text { Death, myocardial infarction, stroke, or any } \\
\text { repeat revascularization }\end{array}$ & 87 (19.9) & $59(13.3)$ & $1.54(1.11-2.14)$ & 0.01 \\
\hline $\begin{array}{l}\text { Death from cardiac cause, myocardial } \\
\text { infarction, stroke, or any repeat } \\
\text { revascularization }\end{array}$ & $78(17.8)$ & $54(12.2)$ & $1.51(1.06-2.13)$ & 0.02 \\
\hline \multicolumn{5}{|l|}{ Bleeding } \\
\hline TIMI major bleeding $\mathbb{}$ & $30(6.8)$ & $132(29.9)$ & $0.20(0.14-0.30)$ & $<0.001$ \\
\hline Fatal bleeding & $3(0.7)$ & $7(1.6)$ & $0.44(0.11-1.68)$ & 0.21 \\
\hline
\end{tabular}

* Percentages are crude rates and are from the intention-to-treat analysis. NA denotes not applicable.

$\dagger$ Hazard ratios and $95 \%$ confidence intervals were assessed for events on the basis of all available follow-up data.

$t P$ values were calculated with the use of the log-rank test on the basis of all available follow-up data.

$\int$ Thrombolysis in Myocardial Infarction (TIMI) major bleeding refers to events that were adjudicated on the basis of TIMI criteria. ${ }^{18}$

mellitus ( $\mathrm{P}=0.06$ for interaction). The benefits of $\mathrm{CABG}$ in this context could be due to the complex and aggressive nature of coronary atherosclerosis in patients with diabetes. The limited antiproliferative effects of everolimus-eluting stents in patients with insulin resistance or deficiency could also have contributed to this difference. ${ }^{25}$

Our study had several limitations. First, this trial was originally powered for the composite 


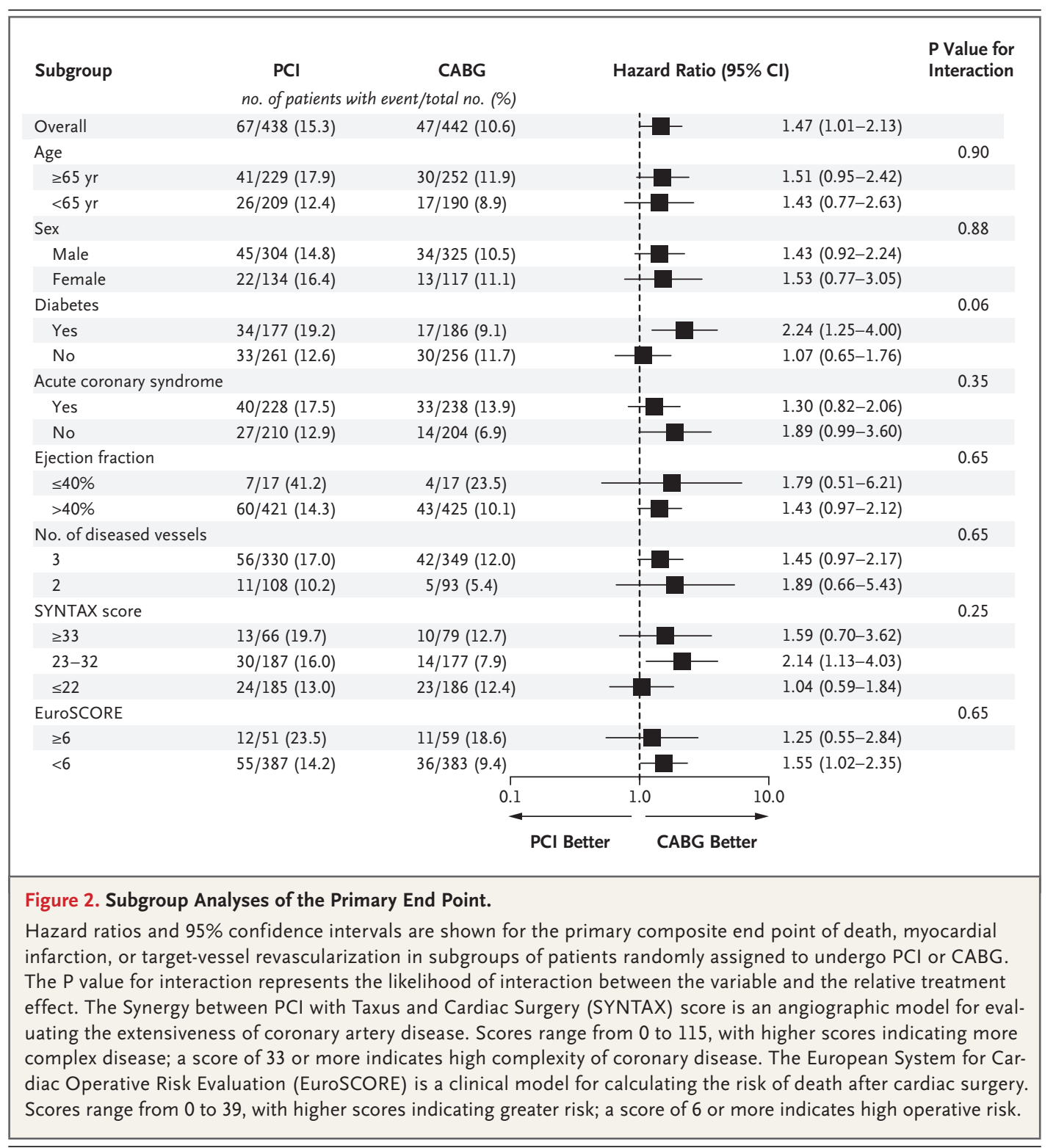

end point of death, myocardial infarction, or target-vessel revascularization. Therefore, it had insufficient power to detect a differential treatment effect between groups for individual end points such as death or myocardial infarction. In addition, the early termination of the trial reduced the statistical power. Second, crossovers, particularly from CABG to PCI, may have introduced a bias, although the results of the astreated analyses were similar to those of the intention-to-treat analyses. Third, owing to the restricted sample size, the results of our subgroup analyses should be considered explora- tory. Fourth, although we tried to enroll all eligible patients, only approximately $20 \%$ of the patients who were screened were finally enrolled. Thus, selection bias may have affected the results. Fifth, the use of some medications differed significantly between the groups. In addition, although we strongly discouraged routine angiographic follow-up, it was performed in some patients and was performed more frequently in the PCI group than in the CABG group. Finally, the fact that the trial included only patients of Asian race could affect the generalizability of the findings. 
In conclusion, in a randomized trial involving patients with multivessel coronary artery disease, PCI with the use of everolimus-eluting stents was not noninferior to CABG with respect to major adverse cardiovascular events at 2 years. In longer-term follow-up, CABG was associated with a lower rate of major adverse cardiovascular events than PCI.
Supported by the CardioVascular Research Foundation and Abbott Vascular and by a grant (HI10C2020) from the Korea Healthcare Technology Research and Development Project, Ministry for Health and Welfare Affairs, South Korea.

Disclosure forms provided by the authors are available with the full text of this article at NEJM.org.

We thank the trial staff members, the other members of the cardiac catheterization laboratories at the participating centers, and the study coordinators for their efforts to collect clinical data and to ensure their accuracy and completeness.

\section{REFERENCES}

1. Serruys PW, Morice MC, Kappetein AP, et al. Percutaneous coronary intervention versus coronary-artery bypass grafting for severe coronary artery disease. N Engl J Med 2009;360:961-72. [Erratum, N Engl J Med 2013;368:584.]

2. Mohr FW, Morice MC, Kappetein AP, et al. Coronary artery bypass graft surgery versus percutaneous coronary intervention in patients with three-vessel disease and left main coronary disease: 5 -year follow-up of the randomised, clinical SYNTAX trial. Lancet 2013;381:62938.

3. Head SJ, Davierwala PM, Serruys PW, et al. Coronary artery bypass grafting vs. percutaneous coronary intervention for patients with three-vessel disease: final five-year follow-up of the SYNTAX trial. Eur Heart J 2014;35:2821-30.

4. Farkouh ME, Domanski M, Sleeper LA, et al. Strategies for multivessel revascularization in patients with diabetes. N Engl J Med 2012;367:2375-84.

5. Deb S, Wijeysundera HC, Ko DT, Tsubota H, Hill S, Fremes SE. Coronary artery bypass graft surgery vs percutaneous interventions in coronary revascularization: a systematic review. JAMA 2013; 310:2086-95.

6. Weintraub WS, Grau-Sepulveda MV, Weiss JM, et al. Comparative effectiveness of revascularization strategies. N Engl J Med 2012;366:1467-76.

7. Sipahi I, Akay MH, Dagdelen S, Blitz A, Alhan C. Coronary artery bypass grafting vs percutaneous coronary intervention and long-term mortality and morbidity in multivessel disease: meta-analysis of randomized clinical trials of the arterial grafting and stenting era. JAMA Intern Med 2014;174:223-30.

8. Hillis LD, Smith PK, Anderson JL, et al. 2011 ACCF/AHA Guideline for Coronary Artery Bypass Graft Surgery: a report of the American College of Cardiology Foundation/American Heart Association Task Force on Practice Guidelines. Circulation 2011;124(23):e652-e735.

9. Windecker S, Kolh P, Alfonso F, et al. 2014 ESC/EACTS Guidelines on myocardial revascularization: the Task Force on Myocardial Revascularization of the European Society of Cardiology (ESC) and the European Association for Cardio-Thoracic Surgery (EACTS), developed with the special contribution of the European Association of Percutaneous Cardiovascular Interventions (EAPCI). Eur Heart J 2014;35: 2541-619.

10. Daemen J, Wenaweser P, Tsuchida K, et al. Early and late coronary stent thrombosis of sirolimus-eluting and paclitaxeleluting stents in routine clinical practice: data from a large two-institutional cohort study. Lancet 2007;369:667-78.

11. Farooq V, Serruys PW, Zhang Y, et al. Short-term and long-term clinical impact of stent thrombosis and graft occlusion in the SYNTAX trial at 5 years: Synergy Between Percutaneous Coronary Intervention with Taxus and Cardiac Surgery trial. J Am Coll Cardiol 2013;62:2360-9.

12. Bangalore S, Kumar S, Fusaro M, et al. Short- and long-term outcomes with drug-eluting and bare-metal coronary stents: a mixed-treatment comparison analysis of 117762 patient-years of follow-up from randomized trials. Circulation 2012;125:2873-91.

13. Gada H, Kirtane AJ, Newman W, et al 5 -Year results of a randomized comparison of XIENCE $\mathrm{V}$ everolimus-eluting and TAXUS paclitaxel-eluting stents: final results from the SPIRIT III trial (clinical evaluation of the XIENCE V everolimus eluting coronary stent system in the treatment of patients with de novo native coronary artery lesions). JACC Cardiovasc Interv 2013;6:1263-6.

14. Park SJ, Kim YH, Park DW, et al. Randomized trial of stents versus bypass surgery for left main coronary artery disease. N Engl J Med 2011;364:1718-27.

15. Mercado N, Wijns W, Serruys PW, et al One-year outcomes of coronary artery bypass graft surgery versus percutaneous coronary intervention with multiple stenting for multisystem disease: a meta-analysis of individual patient data from randomized clinical trials. J Thorac Cardiovasc Surg 2005;130:512-9.

16. Serruys PW, Unger F, Sousa JE, et al. Comparison of coronary-artery bypass surgery and stenting for the treatment of multivessel disease. N Engl J Med 2001; 344:1117-24

17. Cain KC, Lange NT. Approximate case influence for the proportional hazards regression model with censored data. Biometrics 1984;40:493-9.

18. Wiviott SD, Antman EM, Gibson CM, et al. Evaluation of prasugrel compared with clopidogrel in patients with acute coronary syndromes: design and rationale for the TRial to assess Improvement in Therapeutic Outcomes by optimizing platelet InhibitioN with prasugrel Thrombolysis In Myocardial Infarction 38 (TRITON-TIMI 38). Am Heart J 2006;152: 627-35.

19. Laskey WK, Yancy CW, Maisel WH. Thrombosis in coronary drug-eluting stents: report from the meeting of the Circulatory System Medical Devices Advisory Panel of the Food and Drug Administration Center for Devices and Radiologic Health, December 7-8, 2006. Circulation 2007;115:2352-7.

20. Smit Y, Vlayen J, Koppenaal H, et al. Percutaneous coronary intervention versus coronary artery bypass grafting: a metaanalysis. J Thorac Cardiovasc Surg 2014 November 1 (Epub ahead of print).

21. Misfeld M, Brereton RJ, Sweetman EA, Doig GS. Neurologic complications after off-pump coronary artery bypass grafting with and without aortic manipulation: meta-analysis of 11,398 cases from 8 studies. J Thorac Cardiovasc Surg 2011; 142(2):e11-e17.

22. Nasir K, Katz R, Takasu J, et al. Ethnic differences between extra-coronary measures on cardiac computed tomography: Multi-Ethnic Study of Atherosclerosis (MESA). Atherosclerosis 2008;198:104-14. 23. Hannan EL, Racz MJ, Walford G, et al. Long-term outcomes of coronary-artery bypass grafting versus stent implantation. N Engl J Med 2005;352:2174-83.

24. Javaid A, Steinberg DH, Buch AN, et al. Outcomes of coronary artery bypass grafting versus percutaneous coronary intervention with drug-eluting stents for patients with multivessel coronary artery disease. Circulation 2007;116:Suppl:I-200I-206.

25. Stone GW, Rizvi A, Newman W, et al. Everolimus-eluting versus paclitaxeleluting stents in coronary artery disease. N Engl J Med 2010;362:1663-74.

Coppright (c) 2015 Massachusetts Medical Society. 